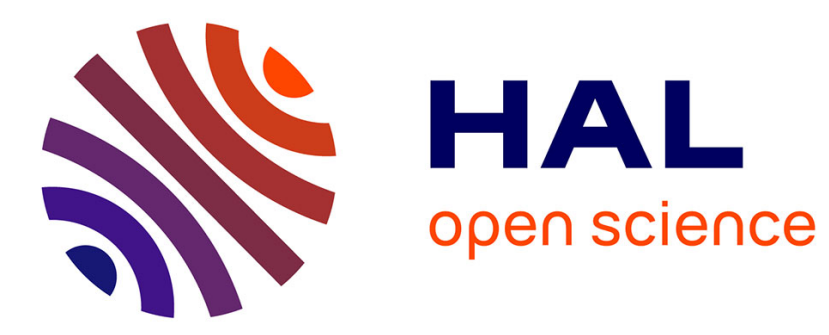

\title{
Triggering mechanism using freely selected sensors for linear time-invariant systems
}

Romain Postoyan, Antoine Girard

\section{To cite this version:}

Romain Postoyan, Antoine Girard. Triggering mechanism using freely selected sensors for linear time-invariant systems. 54th IEEE Conference on Decision and Control, Dec 2015, Osaka, Japan. pp.4812-4817, 10.1109/CDC.2015.7402970 . hal-01204815

\section{HAL Id: hal-01204815 \\ https://hal.science/hal-01204815}

Submitted on 8 Dec 2015

HAL is a multi-disciplinary open access archive for the deposit and dissemination of scientific research documents, whether they are published or not. The documents may come from teaching and research institutions in France or abroad, or from public or private research centers.
L'archive ouverte pluridisciplinaire HAL, est destinée au dépôt et à la diffusion de documents scientifiques de niveau recherche, publiés ou non, émanant des établissements d'enseignement et de recherche français ou étrangers, des laboratoires publics ou privés. 


\title{
Triggering mechanism using freely selected sensors for linear time-invariant systems
}

\author{
Romain Postoyan and Antoine Girard
}

\begin{abstract}
Existing event-triggering sampling techniques for state feedback controllers generally use full state information. This may be a source of important computational and communication costs, particularly if sensors are numerous and/or distributed, as the triggering condition needs to be continuously evaluated. We propose an approach to redesign triggering mechanisms for linear time-invariant systems based on limited sensors information, which we freely select, and an internal scalar dynamic variable. We prove that the resulting closed loop system ensures a uniform global exponential stability property and that a uniform minimal inter-execution time is guaranteed. Besides stabilizability, no additional assumption on the system is needed. Guidelines are provided to derive the redesigned triggering conditions using linear matrix inequalities. We show on an academic example that, using a suitable choice of sensors and parameters, inter-execution times using our approach are comparable to those using triggering mechanisms that monitor the full state of the system.
\end{abstract}

\section{INTRODUCTION}

Event-triggered control is a sampling paradigm, which consists in closing the feedback loop at time instants determined by a state-dependent criterion. The underlying idea is to sample only when this is needed in view of the control objectives. This alternative to periodic sampling is relevant for networked control systems, as it may allow to significantly reduce the controller bandwidth usage, as well as for embedded systems, as it may limit the number controller executions and thus reduce the computational cost associated with the control task. Numerous strategies have been developed in the literature since the pioneering works of [4], [5], [20], see for instance [12], [18] and the references therein. Most of these works concentrate on state-feedback control. In this case, the triggering condition requires to continuously monitor all the states of the system. This may be computationally demanding, in particular when the number of state variables is large. In this case, it would be relevant instead to only consider a subset of the state variables, possibly few of them, and to trigger transmissions only based on these. Such a triggering policy would also be useful for plants with distributed sensors. The implementation of a triggering condition based on the full state vector requires, in this scenario, to resort to a central unit, which continuously collects all the measurements and evaluates the triggering rule, which may be hard to realize in practice. The setup

R. Postoyan is with the Universite de Lorraine, CRAN, UMR 7039 and the CNRS, CRAN, UMR 7039, France, romain.postoyan@univ-lorraine.fr.

A. Girard is with Univ. Grenoble Alpes, LJK, F-38000 Grenoble and CNRS, LJK, F-38000 Grenoble, France, antoine.girardeimag.fr.

Their work was partially supported by the ANR under the grant COMPACS (ANR-13-BS03-0004-02). would be significantly simplified if the triggering mechanism would only work with some output, as it could then be collocated with the associated sensor(s). There is therefore a need for triggering strategies that work with limited sensors information. The purpose of this paper is to address this problem for linear time-invariant systems.

We first design a state-feedback event-triggered controller, thus assuming that the full state can be measured. The technique in [20] can be used at this step, but others can be applied. Afterwards, we select some of the states of the plant to form an output vector. We are free to select this output as we wish: no detectability condition is needed. In practice, we would typically take a small number of sensors compared to the dimension of the state in order to reduce the computation complexity of the triggering mechanism, or we would select sensors which are co-located, to facilitate the implementation. We then redesign the original triggering condition so that it only depends on the selected output, as well as on the value of the full state vector at the last transmission instant, and an additional scalar variable. The latter is introduced by design, and is easy to implement. To use an auxiliary variable to define the triggering rule is also done in [6], [9], [18] in different contexts. The control input is still given by the state-feedback law but the triggering condition is now output-based. The setup works as follows. The triggering mechanism is collocated with the selected sensors, and it continuously evaluates the redesigned triggering condition. When the triggering rule is violated, a request is sent to all the sensors to transmit their current data to the controller and to the triggering mechanism. The updated control input is then sent to the actuators. We ignore the possible time-delay between the time at which the triggering mechanism requests the value of the whole state vector and the time at which the updated control input is applied to the plant; noting that this delay can be analysed by following similar lines as in [20].

We assume that the original state-feedback event-triggered controller ensures both a uniform global exponential stability property and the existence of a global uniform lower bound on the inter-transmission times (thus ruling out Zeno phenomenon). We prove that these two properties still hold with the redesigned triggering rule. We also explain how to tune the triggering parameters using linear matrix inequalities to reduce its potential conservatism. Simulation results are provided for the academic example considered in [20]. We show that the proposed redesigned technique generates a similar amount of transmissions compared to the statefeedback event-triggered controller of [20], while it only 
continuously monitors a single state variable.

The proposed strategy is related to works on outputfeedback event-triggered control (see e.g., [1], [7], [21]) and on distributed event-triggered control (see e.g., [13], [16], [17], [22]). However, it differs from these studies, as the control input is given by a state-feedback law and the triggering condition depends on the value of the full state at the last transmission, which is excluded in the aforementioned studies. In that way, it is expected to generate less transmissions as if the feedback law would be outputbased or distributed, as done in the aforementioned works. Furthermore, contrary to observer-based solutions, we do not resort to state estimates to define the triggering condition, which helps reducing the computational complexity of the scheme.

The paper is organised as follows. Preliminaries are given in Section II, and the problem is stated in Section III. The redesign of the triggering condition and its analysis are respectively presented in Sections IV and V. Simulations results on a numerical example are provided in Section VII, and Section VIII concludes the paper. The proofs are omitted for the sake of brevity.

\section{PRELIMINARIES}

Let $\mathbb{R}:=(-\infty, \infty), \mathbb{R}_{\geq 0}:=[0, \infty), \mathbb{Z}_{\geq 0}:=\{0,1,2, \ldots\}$, and $\mathbb{Z}_{>0}:=\{1,2, \ldots\}$. A function $\gamma: \mathbb{R}_{\geq 0} \rightarrow \mathbb{R}_{\geq 0}$ is of class $\mathcal{K}_{\infty}$ if it is continuous, strictly increasing, zero at zero and unbounded. Let $(x, y) \in \mathbb{R}^{n+m},(x, y)$ stands for $\left[x^{\mathrm{T}}, y^{\mathrm{T}}\right]^{\mathrm{T}}$. The notation $\mathbb{I}$ denotes the identity matrix, whose dimensions depend on the context. In matrices, the symbol $\star$ stands for the symmetric block component. Let $P \in \mathbb{R}^{n \times n}$ be a real, symmetric matrix, we respectively denote its maximum and minimum eigenvalues by $\lambda_{\max }(P)$ and $\lambda_{\min }(P)$. We denote the trace of a square matrix $A$ by $\operatorname{tr}(A)$. The lemma below is used several times in the forthcoming developments.

Lemma 1: For any vectors $v, w$ and any symmetric, positive definite matrix $E, 2 v^{\mathrm{T}} w \leq v^{\mathrm{T}} E v+w^{\mathrm{T}} E^{-1} w$.

We study hybrid systems of the form below [10]

$$
\left\{\begin{aligned}
x \in \mathcal{C} & \dot{x}=F(x) \\
x \in \mathcal{D} & x^{+}=G(x),
\end{aligned}\right.
$$

where $x \in \mathbb{R}^{n}$ is the state, $F$ is the flow map, $G$ is the jump map, $\mathcal{C}$ is the flow set and $\mathcal{D}$ is the jump set. We assume that system (1) satisfies the hybrid basic conditions (see Assumption 6.5 in [10]), which will be the case in the following. We recall some definitions related to [10]. A subset $E \subset \mathbb{R}_{\geq 0} \times \mathbb{Z}_{\geq 0}$ is a hybrid time domain if for all $(T, J) \in E$, $E \cap([0, T] \times\{0, \ldots, J\})=\underset{j \in\{0,1, \ldots, J-1\}}{\bigcup}\left(\left[t_{j}, t_{j+1}\right], j\right)$ for some finite sequence of times $0=t_{0} \leq t_{1} \leq \ldots \leq t_{J}$. A function $\phi: E \rightarrow \mathbb{R}^{n}$ is a hybrid arc if $E$ is a hybrid time domain and if for each $j \in \mathbb{Z}_{\geq 0}, t \mapsto \phi(t, j)$ is locally absolutely continuous on $\{t:(t, \bar{j}) \in E\}$. The hybrid arc $\phi: \operatorname{dom} \phi \rightarrow \mathbb{R}^{n}$ is a solution to (1) if: (i) $\phi(0,0) \in \mathcal{C} \cup \mathcal{D}$; (ii) for any $j \in \mathbb{Z}_{\geq 0}, \phi(t, j) \in \mathcal{C}$ and $\frac{d}{d t} \phi(t, j)=F(\phi(t, j))$ for almost all $t \in I^{j}:=\{t:(t, j) \in \operatorname{dom} \phi\}$; (iii) for every $(t, j) \in \operatorname{dom} \phi$ such that $(t, j+1) \in \operatorname{dom} \phi, \phi(t, j) \in \mathcal{D}$ and $\phi(t, j+1)=G(\phi(t, j))$. A solution $\phi$ to (1) is:

- nontrivial if dom $\phi$ contains at least two points;

- maximal if it cannot be extended;

- complete if dom $\phi$ is unbounded.

Note that a solution may be maximal but not complete.

\section{PROBlem Statement}

Consider the linear time-invariant system

$$
\dot{x}=A x+B u
$$

where $x \in \mathbb{R}^{n_{x}}$ is the state, $u \in \mathbb{R}^{n_{u}}$ is the control input, $A$ and $B$ are real matrices of appropriate dimensions such that the pair $(A, B)$ is stabilizable, and $n_{x}, n_{u} \in \mathbb{Z}_{>0}$. We design a state-feedback law of the form

$$
u=K x
$$

where $K$ is a real matrix such that $A+B K$ is Hurwitz. In that way, the origin of the closed-loop system (2)-(3) is uniformly globally exponentially stable.

We investigate the scenario where controller (3) is implemented on a digital platform using zero-order-hold devices. As a consequence, the closed-loop system becomes

$$
\dot{x}=A x+B K \hat{x}
$$

where $\hat{x}$ is defined as, for any $i \in \mathcal{I} \subseteq \mathbb{Z}_{\geq 0}$,

$$
\hat{x}(t)=x\left(t_{i}\right) \quad \text { for almost all } t \in\left[t_{i}, t_{i+1}\right]
$$

and $t_{i}, i \in \mathcal{I}$, denotes the sequence of time instants at which the control input is updated. We focus on the case where the sequence $t_{i}, i \in \mathcal{I}$, is determined by an event-triggering condition. We introduce the sampling-induced error $e:=$ $\hat{x}-x \in \mathbb{R}^{n_{x}}$ and we model the overall dynamics as a hybrid system using the formalism of [10] for which a jump corresponds to an update of the control input (like in e.g., [7], [8], [18], [19])

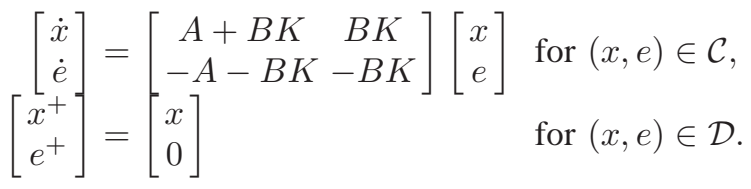

The flow set $\mathcal{C}$ and the jump set $\mathcal{D}$ are defined according to the triggering condition. Solutions to (6) flows when $(x, e) \in \mathcal{C}$ and a jump occurs when they enter in $\mathcal{D}$, which corresponds to the region of the state space where the triggering condition is violated ${ }^{1}$.

Various techniques are available in the literature to design the triggering condition (i.e. the sets $\mathcal{C}$ and $\mathcal{D}$ ) so that stability is preserved under sampling. For instance in Section II.B in [12], the triggering rule is constructed as follows. Let $P, Q$ be real, symmetric, positive definite matrices such that $(A+B K)^{\mathrm{T}} P+P(A+B K)=-Q$ (such matrices always exist since $A+B K$ is Hurwitz, see Theorem 4.6 in [14]) and

\footnotetext{
${ }^{1}$ When $(x, e) \in \mathcal{C} \cap \mathcal{D}$, the solution can either jump or flow, the latter is only possible if flowing keeps $(x, e)$ in $\mathcal{C}$.
} 
define $V(x):=x^{\mathrm{T}} P x$ for any $x \in \mathbb{R}^{n_{x}}$. For any $(x, e) \in$ $\mathbb{R}^{2 n_{x}}$

$$
\langle\nabla V(x),(A+B K) x+B K e\rangle=-x^{\mathrm{T}} Q x+2 x^{\mathrm{T}} P B K e .
$$

Consequently, when

$$
2 x^{\mathrm{T}} P B K e \leq \sigma x^{\mathrm{T}} Q x,
$$

with $\sigma \in(0,1)$,

$$
\langle\nabla V(x), A x+B K \hat{x}\rangle \leq-(1-\sigma) x^{\mathrm{T}} Q x,
$$

and the strict decrease of the Lyapunov function $V$ along the solutions to the $x$-system (outside the origin) is preserved. In this case, the sets $\mathcal{C}$ and $\mathcal{D}$ in (6) are given by

$$
\begin{aligned}
\mathcal{C} & =\left\{(x, e): 2 x^{\mathrm{T}} P B K e \leq \sigma x^{\mathrm{T}} Q x\right\}, \\
\mathcal{D} & =\left\{(x, e): 2 x^{\mathrm{T}} P B K e \geq \sigma x^{\mathrm{T}} Q x\right\} .
\end{aligned}
$$

The condition in (8) has a quadratic form, and we can rewrite it as (see (8) in [12])

$$
\left[\begin{array}{l}
e \\
x
\end{array}\right]^{\mathrm{T}}\left[\begin{array}{cc}
0 & \star \\
P B K & -\sigma Q
\end{array}\right]\left[\begin{array}{l}
e \\
x
\end{array}\right] \leq 0 .
$$

This is a particular type of quadratic event-triggering condition, others are possible, see Section II.B in [11]. In this note, we thus concentrate on triggering conditions of the general form

$$
\begin{aligned}
& \mathcal{C}=\left\{(x, e) \in \mathbb{R}^{2 n_{x}}:\left[\begin{array}{l}
e \\
x
\end{array}\right]^{\mathrm{T}} S\left[\begin{array}{l}
e \\
x
\end{array}\right] \leq 0\right\} \\
& \mathcal{D}=\left\{(x, e) \in \mathbb{R}^{2 n_{x}}:\left[\begin{array}{l}
e \\
x
\end{array}\right]^{\mathrm{T}} S\left[\begin{array}{l}
e \\
x
\end{array}\right] \geq 0\right\},
\end{aligned}
$$

where $S$ is a real matrix, which we write as $S=\left[\begin{array}{cc}S_{1} & \star \\ S_{2} & -S_{3}\end{array}\right]$ with $S_{3} \in \mathbb{R}^{n_{x} \times n_{x}}$. We assume that the matrix $S$ is designed in such a way that following assumption is verified.

Assumption 1: The following holds.

(i) The matrix $S_{3}$ is symmetric and positive definite.

(ii) There exist a real, symmetric, and positive definite matrix $P \in \mathbb{R}^{n_{x} \times n_{x}}$ and $\alpha>0$ such that, for any $(x, e) \in \mathbb{R}^{2 n_{x}}$,

$$
\begin{aligned}
& (x, e) \in \mathcal{C} \\
& \quad \Rightarrow\langle\nabla V(x),(A+B K) x+B K e\rangle \leq-\alpha|x|^{2},
\end{aligned}
$$

where $V(x)=x^{\mathrm{T}} P x$ for any $x \in \mathbb{R}^{n_{x}}$.

Item (i) of Assumption 1 is justified by the following arguments. First, we can always write $S$ in (12) such that $S_{3}$ is symmetric. Second, $S_{3}$ must be positive definite to avoid Zeno phenomenon (outside the origin). Indeed, after a jump, $\left[\begin{array}{l}e^{+} \\ x^{+}\end{array}\right]^{\mathrm{T}} S\left[\begin{array}{l}e^{+} \\ x^{+}\end{array}\right]=\left[\begin{array}{l}0 \\ x\end{array}\right]^{\mathrm{T}} S\left[\begin{array}{l}0 \\ x\end{array}\right]=-x^{T} S_{3} x$ according to (6). If $S_{3}$ would not be positive definite, there would exist $x_{*} \in \mathbb{R}^{n_{x}} \backslash\{0\}$ such that $-x_{*}^{T} S_{3} x_{*} \geq 0$, which implies that $\left(x_{*}, 0\right) \in \mathcal{D}$ in view of (12). As a consequence, any solution reaching $\left(x_{*}, 0\right)$ may then jump instantaneously infinitely many times. To have $S_{3}$ positive definite avoids this issue. We will show later that item (i) of Assumption 1 not only prevents Zeno phenomenon but it also ensures the existence of a uniform dwell-time outside the origin ${ }^{2}$. Item (ii) of Assumption 1 means that the triggering condition is designed to ensure the strict decrease of a quadratic Lyapunov function $V$. Examples of matrices $S$, which fulfill Assumption 1, include the one in (11) (in which case $S_{3}=$ $\sigma Q, P$ is given above (7) and $\left.\alpha=(1-\sigma) \lambda_{\min }(Q)\right)$, others possibilities can be found in Section II.B in [11].

The objective of this work is to redesign the triggering rule in (12) so that it no longer depends on the full state of the system but only on a given output, which we are free to select, the value of the state vector at the last transmission instant, i.e. $\hat{x}$, and an auxiliary scalar variable we will design.

\section{REDESIGN OF THE TRIGGERING CONDITION}

\section{A. Principle}

As a preliminary step, we rewrite the quadratic term in (12) in terms of $(e, \hat{x})$ (instead of $(e, x)$ ). We use for that purpose the fact that $x=\hat{x}-e$,

$$
\left[\begin{array}{l}
e \\
\hat{x}
\end{array}\right]^{\mathrm{T}}\left[\begin{array}{cc}
\mathbb{I} & 0 \\
-\mathbb{I} & \mathbb{I}
\end{array}\right]^{\mathrm{T}} S\left[\begin{array}{cc}
\mathbb{I} & 0 \\
-\mathbb{I} & \mathbb{I}
\end{array}\right]\left[\begin{array}{l}
e \\
\hat{x}
\end{array}\right]=\left[\begin{array}{l}
e \\
\hat{x}
\end{array}\right]^{\mathrm{T}} \widehat{S}\left[\begin{array}{l}
e \\
\hat{x}
\end{array}\right],
$$

with

$$
\widehat{S}:=\left[\begin{array}{cc}
\mathbb{I} & 0 \\
-\mathbb{I} & \mathbb{I}
\end{array}\right]^{\mathrm{T}} S\left[\begin{array}{cc}
\mathbb{I} & 0 \\
-\mathbb{I} & \mathbb{I}
\end{array}\right] .
$$

We introduce $y=C x$ to denote the output of system (2) we choose to continuously monitor. The real matrix $C$ is designed and can be arbitrarily selected (it does not have to be such that the pair $(A, C)$ is detectable). Since we assume that we know the value of $y$ at any time instant, we also know $e_{y}:=C e$ at any time instant. We then propose to upper bound (14) as follows

$$
\left[\begin{array}{l}
e \\
\hat{x}
\end{array}\right]^{\mathrm{T}} \widehat{S}\left[\begin{array}{l}
e \\
\hat{x}
\end{array}\right] \leq\left[\begin{array}{c}
C e \\
\hat{x}
\end{array}\right]^{\mathrm{T}} M\left[\begin{array}{c}
C e \\
\hat{x}
\end{array}\right]+e^{\mathrm{T}} R e,
$$

where $M=\left[\begin{array}{cc}M_{1} & \star \\ M_{2} & -M_{3}\end{array}\right]$ is a real, symmetric matrix with $M_{3} \in \mathbb{R}^{n_{x} \times n_{x}}$ positive definite, and $R$ is a real, symmetric, and positive definite matrix. Note that (16) can always be guaranteed according to the lemma below.

Lemma 2: If item (i) of Assumption 1 holds, then there exist real, symmetric matrices $M$ and $R$ such that (16) is verified with $M_{3}$ and $R$ positive definite.

The first term on the right hand-side of (16) depends on $\mathrm{Ce}$, which we continuously monitor, and on $\hat{x}$, which is available as it corresponds to the value of the state at the last transmission instant. On the other hand, we do not want the new triggering condition to depend on the term $e^{\mathrm{T}} R e$ in (16). We thus propose to upper-bound $e^{\mathrm{T}} R e$ by a variable $p \in \mathbb{R}$, whose dynamics is designed in the sequel. In that way, we aim at enforcing the condition below on flows

$$
\left[\begin{array}{c}
C e \\
\hat{x}
\end{array}\right]^{\mathrm{T}} M\left[\begin{array}{c}
C e \\
\hat{x}
\end{array}\right]+p \leq 0 .
$$

\footnotetext{
${ }^{2}$ See Remark 4 in Section V-A.
} 
We model the obtained system as follows

$$
\left.\begin{array}{rl}
\dot{x} & =(A+B K) x+B K e \\
\dot{e} & =-(A+B K) x-B K e \\
\dot{p} & =f(p, \hat{x}, y) \\
x^{+} & =x \\
e^{+} & =0 \\
p^{+} & =g(p, \hat{x}, y)
\end{array}\right\} \quad(x, e, p) \in \widehat{\mathcal{C}}
$$

where $f$ and $g$ have to be designed and the flow and the jump sets are defined in (19), where $W(e):=e^{\mathrm{T}} R e$ for any $e \in \mathbb{R}^{n_{x}}$.

The fact that $p$ upper-bounds $W(e)$, i.e. $e^{\mathrm{T}} R e$, is somehow enforced by the definitions of $\widehat{\mathcal{C}}$ and $\widehat{\mathcal{D}}$ in (19). However, this condition does not need to be implemented in practice because of the way we construct the dynamics of $p$ in the following. Indeed, we will show that the variable $p$ always upper-bounds $W(e)$ along the solutions to (18), except, possibly, at the initial time, which justifies the definitions of $\widehat{\mathcal{C}}$ and $\widehat{\mathcal{D}}$ in (19) that enforce the 'right' initialization of $p$.

\section{B. Design}

1) Matrix $R$ : We select the matrix $R$ in (16) such that there exist real matrices $L, \bar{R}$ with $\bar{R}$ symmetric and positive semi-definite, and a constant $\lambda \in \mathbb{R}$, which verify

$$
(A-L C)^{\mathrm{T}} R+R(A-L C) \leq \lambda R-C^{T} \bar{R} C .
$$

This condition is always satisfied by selecting $\lambda$ sufficiently large and setting $\bar{R}=0$ for instance. Inequality (20) is used to construct the dynamics of the variable $p$ on flows (namely $f$ in (18)).

2) Dynamics of $p$ : We rewrite the dynamics of the $e$ variable on flows as, using $x=\hat{x}-e$,

$$
\dot{e}=-(A+B K) x-B K e=D \hat{x}+A e,
$$

where $D:=-A-B K$. Consequently, for any $(x, e) \in \mathbb{R}^{2 n_{x}}$,

$$
\begin{aligned}
\langle\nabla W(e), A e+D \hat{x}\rangle & =\langle\nabla W(e),(A-L C) e+D \hat{x}+L C e\rangle \\
& =\left\langle\nabla W(e),(A-L C) e+D \hat{x}+L e_{y}\right\rangle
\end{aligned}
$$

where $L$ comes from (20), and recall that $e_{y}=C e$. Thus, using (20) and Lemma 1 with $v=R e, w=D \hat{x}+L e_{y}$ and $E=\alpha R^{-1}$ where $\alpha>0$,

$$
\begin{aligned}
\langle\nabla W(e), A e+D \hat{x}\rangle \leq & \lambda W(e)-e_{y}^{\mathrm{T}} \bar{R} e_{y}+2 e^{\mathrm{T}} R\left(D \hat{x}+L e_{y}\right) \\
\leq & \lambda W(e)-e_{y}^{\mathrm{T}} \bar{R} e_{y}+\alpha e^{\mathrm{T}} R e \\
& +\frac{1}{\alpha}\left(D \hat{x}+L e_{y}\right)^{\mathrm{T}} R\left(D \hat{x}+L e_{y}\right) \\
= & (\lambda+\alpha) W(e)-e_{y}^{\mathrm{T}} \bar{R} e_{y} \\
& +\frac{1}{\alpha}\left(D \hat{x}+L e_{y}\right)^{\mathrm{T}} R\left(D \hat{x}+L e_{y}\right) .
\end{aligned}
$$

In view of (18) and (23), we define the flow map and the jump map for the variable $p$ in (18) as

$$
\begin{aligned}
f(p, \hat{x}, y):=\quad & (\lambda+\alpha) p-e_{y}^{\mathrm{T}} \bar{R} e_{y} \\
& +\frac{1}{\alpha}\left(D \hat{x}+L e_{y}\right)^{\mathrm{T}} R(D)= \\
g(p, \hat{x}, y):= & 0 .
\end{aligned}
$$

The dynamics of $p$ only involve $p, e_{y}$ and $\hat{x}$, which are all continuously available to the triggering mechanism.
Remark 1: The construction of the dynamics of $p$ on flows is inspired by works on norm-estimators, in particular [15]. However, the $p$-system cannot be considered as a normestimator of the $e$-system as it does not a priori satisfy the input-to-state stability property stated in item (i) of Definition 2.4 in [15]. This comes from the fact that $\lambda$ in (20) is not necessarily strictly negative (in which case the pair $(A, C)$ would need to be detectable). We can relax the requirement that $\lambda$ is strictly negative because that is not needed to upperbound $W(e)$ and because we know the value of $W(e)$ after each jump instant (it is equal to zero according to (18)). We therefore tolerate the $p$-system to have unstable dynamics on flows. This instability is compensated by the reset of $p$ at 0 at each jump. We will show in Theorem 1 that the $p$ component of the solutions are bounded, more precisely, we will guarantee that $(x, e, p)=0$ is uniformly globally stable (see Definition 3.6 in [10]).

Remark 2: The non-positive term $-C^{T} \bar{R} C$ in (20) may be useful to reduce the conservatism of the upper-bound of $W(e)$ given by $p$.

Remark 3: When $\bar{R}=0, L=0, M_{1}=0, M_{2}=0$, the triggering condition in (19) is independent of $e_{y}$. In this case, we do not need to continuously monitor the output $y$, as only the value of $\hat{x}$ is needed to decide the next triggering instant: we have a self-triggered-like policy (see e.g., [2], [3], [23], [24]).

\section{ANALYSIS}

\section{A. Existence of a uniform dwell-time}

The following proposition states that the triggering condition defined in (19) guarantees the existence of a uniform minimum amount of (ordinary) time between two successive transmissions (outside the origin).

Proposition 1: Consider system (18), (19) and suppose item (i) of Assumption 1 holds. There exists $\theta>0$ such that for any solution $(x, e, p)$ and any $(s, i),(t, j) \in \operatorname{dom}(x, e, p)$ with $s+i \leq t+j,(x(t, j), e(t, j), p(t, j)) \neq 0$ implies $j-i \leq \frac{t-\bar{s}}{\theta}+1$

The constant $\theta$ in Proposition 1 is a uniform global lower bound on the inter-transmissions intervals. The existence of this dwell-time is not ensured at the origin for system (18), (19) in Proposition 1. Nevertheless, when a solution is at the origin, it can either keep flowing forever, keep instantaneously jumping infinitely many times, or do any intermediate behaviour as it lies in the intersection of the flow and the jump set and flowing keeps the solution in $\widehat{\mathcal{C}}$. This means that we can transmit whenever we want in this case in practice, and the existence of dwell-time can therefore be enforced. A similar observation is made in Section IV.B in [18].

Remark 4: The redesigned triggering conditions upperbounds the one in (12) in view of (16) and the fact that $W(e) \leq p$. We deduce that the existence of a global uniform dwell-time (outside the origin) for system (18), (19) ensures the existence of such a time for system (6), (12). 


$$
\begin{aligned}
& \widehat{\mathcal{C}}=\left\{(x, e, p):\left[\begin{array}{c}
C e \\
\hat{x}
\end{array}\right]^{\mathrm{T}} M\left[\begin{array}{c}
C e \\
\hat{x}
\end{array}\right]+p \leq 0 \text { and } W(e) \leq p\right\} \\
& \widehat{\mathcal{D}}=\left\{(x, e, p):\left[\begin{array}{c}
C e \\
\hat{x}
\end{array}\right]^{\mathrm{T}} M\left[\begin{array}{c}
C e \\
\hat{x}
\end{array}\right]+p \geq 0 \text { and } W(e) \leq p\right\} .
\end{aligned}
$$

\section{B. Stability}

The theorem below shows that stability is preserved with the redesigned triggering condition.

Theorem 1: Consider system (18), (19) and suppose Assumption 1 is verified. The following holds.

(i) There exist $c_{1} \geq 1$ and $c_{2}>0$ such that for any solution, $|x(t, j)| \leq c_{1} \exp \left(-c_{2}(t+j)\right)|x(0,0)|$ for any $(t, j)$ in the domain of the solution.

(ii) There exists $\rho \in \mathcal{K}_{\infty}$ such that for any solution, $\quad|(x(t, j), e(t, j), p(t, j))| \leq$ $\exp \left(-c_{2} j\right) \rho(|(x(0,0), e(0,0), p(0,0))|)$ for any $(t, j)$ in the domain of the solution.

(iii) Any maximal solution is complete.

Item (i) of Theorem 1 means that the uniform global exponential stability property of system (6), (12), guaranteed through the satisfaction of item (ii) of Assumption 1, is preserved with the redesigned triggering rule. Item (ii) of Theorem 1 implies that $(x, e, p)=0$ is uniformly globally stable (see Definition 3.6 in [10]), and that this stability property becomes asymptotic when the maximal solutions have an unbounded time domain in $j$, which may not be the case in general. Item (iii) of Theorem 1 confirms that $p$ is an appropriate upper-bound of $W(e)$; if it would not be the case, some maximal solutions would not be complete.

\section{Guidelines}

The approach described in Section IV consists in suitably upper-bounding the original triggering condition in (12). To this purpose, we first use the inequality in (16) and we then construct the dynamics of the variable $p$ to upper-bound $e^{\mathrm{T}} R e$, see Section IV-B.2. In this section, we explain how to optimize the selection of the triggering condition parameters to reduce the potential conservatism induced by these two steps. We first treat them separately, and we then present the overall optimization problem.

\section{A. Inequality (16)}

Given the matrix $C$, we select the real, symmetric matrices $M$ and $R$ with $M_{3}$ and $R$ positive definite, such that they minimize

$$
\operatorname{tr}\left(\left[\begin{array}{cc}
C^{\mathrm{T}} & 0 \\
0 & \mathbb{I}
\end{array}\right] M\left[\begin{array}{cc}
C & 0 \\
0 & \mathbb{I}
\end{array}\right]+\left[\begin{array}{cc}
R & 0 \\
0 & 0
\end{array}\right]-\widehat{S}\right),
$$

which is equivalent to minimizing

$$
\operatorname{tr}\left(\left[\begin{array}{cc}
C^{\mathrm{T}} & 0 \\
0 & \mathbb{I}
\end{array}\right] M\left[\begin{array}{cc}
C & 0 \\
0 & \mathbb{I}
\end{array}\right]+\left[\begin{array}{cc}
R & 0 \\
0 & 0
\end{array}\right]\right) .
$$

This problem can be efficiently solved using standard linear matrix inequalities (LMI) solvers.

\section{B. Dynamics of $p$}

There are two possible sources of conservatism in Section IV-B.2, and the first one is (20). One way to limit it is to construct the matrices $R, \bar{R}, L$ with $\bar{R}$ symmetric and positive semi-definite, to minimize

$$
\operatorname{tr}\left(\lambda R-C^{T} \bar{R} C-(A-L C)^{\mathrm{T}} R-R(A-L C)\right) .
$$

This problem is linear in the variables $R, \bar{R}$ and $Z=R L$ for given $C$ and $\lambda$, and it can therefore be solved using LMI solvers.

On the other hand, the developments in (23) may also be a source of conservatism. To vary the value of $\alpha$ may be helpful here.

\section{Optimization problem}

There are two criteria to minimize, namely (26) and (27). We choose to formulate this multi-objective problem using a weighted sum. In that way, for given $C, \lambda \in \mathbb{R}_{\geq 0}, \alpha>0$ and weighths $\chi_{1}, \chi_{2} \geq 0$, we aim at solving

$$
\begin{aligned}
& \min \chi_{1} \operatorname{tr}\left(\left[\begin{array}{cc}
C^{\mathrm{T}} & 0 \\
0 & \mathbb{I}
\end{array}\right] M\left[\begin{array}{cc}
C & 0 \\
0 & \mathbb{I}
\end{array}\right]+\left[\begin{array}{cc}
R & 0 \\
0 & 0
\end{array}\right]\right) \\
& \quad+\chi_{2} \operatorname{tr}\left(\lambda R-C^{T} \bar{R} C-(A-L C)^{\mathrm{T}} R-R(A-L C)\right) \\
& \text { subject to }(16),(20) \text { and } \\
& \left\{\begin{array}{l}
M=M^{\mathrm{T}} \text { with } M_{3}>0 \\
R=R^{\mathrm{T}}>0 \\
\bar{R}=\bar{R}^{\mathrm{T}} \geq 0 .
\end{array}\right.
\end{aligned}
$$

The scalars $\lambda, \alpha, \chi_{1}, \chi_{2}$ need to be fixed to solve the problem above. A heuristic line search can be done off-line, as we did in Section VII on a numerical example. Another important question is how to select the matrix $C$ and thus the states that we continuously monitor. First, we may be constrained by practical requirements. If several options are possible, there are always a finite number of these and we may test all of them off-line, as done on an example in Section VII.

\section{ILLUSTRATIVE EXAMPLE}

We consider the example studied in Section V in [20], namely

$$
\left[\begin{array}{l}
\dot{x}_{1} \\
\dot{x}_{2}
\end{array}\right]=\left[\begin{array}{ll}
0 & 1 \\
2 & 3
\end{array}\right]\left[\begin{array}{l}
x_{1} \\
x_{2}
\end{array}\right]+\left[\begin{array}{l}
0 \\
1
\end{array}\right] u
$$

which we stabilize using the feedback law $u=x_{1}-4 x_{2}$. We design the triggering condition (12) with $S$ as in (11), where $P=\left[\begin{array}{cc}1 & \frac{1}{4} \\ \frac{1}{4} & 1\end{array}\right], Q=\left[\begin{array}{cc}\frac{1}{2} & \frac{1}{4} \\ \frac{1}{4} & \frac{3}{2}\end{array}\right]$ and $\sigma=0.5$.

We then apply the method described in Section IV to obtain the desired redesigned triggering condition. We do this for both $y=x_{1}$ and $y=x_{2}$. In each case, we 


\begin{tabular}{c|c|ccc|ccc} 
& $(11)$ & $y=x_{1}$ & & $y=x_{2}$ \\
& & $\left(\chi_{1}, \chi_{2}\right)=(1,0)$ & $\left(\chi_{1}, \chi_{2}\right)=\left(\frac{1}{2}, \frac{1}{2}\right)$ & $\left(\chi_{1}, \chi_{2}\right)=(0,1)$ & $\left(\chi_{1}, \chi_{2}\right)=(1,0)$ & $\left(\chi_{1}, \chi_{2}\right)=\left(\frac{1}{2}, \frac{1}{2}\right)$ & $\left(\chi_{1}, \chi_{2}\right)=(0,1)$ \\
& & $\lambda=10$ & $\lambda=10$ & $\lambda=10$ & $\lambda=-5$ & $\lambda=0$ & $\lambda=-10$ \\
\hline$\tau_{\text {avg }}$ & 0.4683 & 0.0376 & 0.0900 & 0.0530 & 0.4892 & 0.4137 \\
$\tau_{\text {min }}$ & 0.1895 & 0.0137 & 0.0474 & 0.0226 & 0.1263 & 0.1764 \\
TABLE I \\
VALUES OF $\tau_{\text {AVG AND }} \tau_{\text {min }}$.
\end{tabular}

synthesize the matrices $M, R, \bar{R}, L$ by solving (28) for fixed values of $\lambda, \alpha, \chi_{1}, \chi_{2}$. We have run simulations for 10 values of $\left(x_{1}(0,0), x_{2}(0,0)\right)$ uniformly distributed along the unit disk of $\mathbb{R}^{2},(e(0,0), p(0,0))=0$ and for 10 seconds. We have selected $\alpha=10$ and we have varied $\lambda$ within $\{-15,-10,-5,0,5,10,15\}$. Note that we can take $\lambda<0$ as the system is detectable with respect to each output. The values of the average inter-transmission times, denoted $\tau_{\text {avg }}$ and which serves to evaluate the amount of transmissions, and of the minimum inter-transmission times, denoted $\tau_{\min }$, over all simulations are provided in Table I (only the values of $\lambda$, for which the largest value of $\tau_{\text {avg }}$ was observed, are reproduced in this table for each pair $\left(\chi_{1}, \chi_{2}\right)$ ). Comparable values of $\tau_{\text {avg }}$ and of $\tau_{\min }$ are obtained with the original event-triggering condition (11) and with the proposed redesigned policy for $y=x_{2}$. We even have a larger value of $\tau_{\text {avg }}$ with the redesigned technique when $y=x_{2},\left(\chi_{1}, \chi_{2}\right)=(1,0)$ and $\lambda=-5$. Starting from the same initial condition, the first inter-transmission interval will be larger with (11) than with the redesigned triggering rule. However, afterwards, each strategy generates different solutions, which evolve in different regions of the state space. It may therefore be the case that the solutions given by the redesigned policy enter in a region where less transmissions are generated. On the other hand, to take $y=x_{1}$ generates much more transmissions, which indicates that the choice of the monitored output is important when redesigning the triggering condition.

\section{CONCLUSIONS}

We have presented a method to design a triggering condition which works with limited sensors information for linear time-invariant systems. The idea is to start from a known state-feedback event-triggered controller and to redesign its triggering rule so that it no longer depends on the full state vector. In future work, we will show that the method applies to other types of triggering conditions, such as those involving timers (see e.g., [1], [8], [21]) and for systems affected by exogenous disturbances.

\section{REFERENCES}

[1] M. Abdelrahim, R. Postoyan, J. Daafouz, and D. Nešić. Stabilization of nonlinear systems using event-triggered output feedback laws. In International Symposium on Mathematical Theory of Networks and Systems, Groningen, The Netherlands, 2014.

[2] A. Anta and P. Tabuada. To sample or not to sample: self-triggered control for nonlinear systems. IEEE Transactions on Automatic Control, 55(9):2030-2042, 2010.

[3] A. Anta and P. Tabuada. Exploiting isochrony in self-triggered control. IEEE Transactions on Automatic Control, 57(4):950-962, 2012.
[4] K.E. Årzén. A simple event-based PID controller. In Proceedings of the 14th IFAC World Congress, Beijing, China, volume 18, pages 423-428, 1999.

[5] K.J. Aström and B.M. Bernhardsson. Comparison of periodic and event based sampling for first-order stochastic systems. In Proceedings of the 14th IFAC World congress, Beijing, China, volume 11, pages 301-306, 1999.

[6] V.S. Dolk, D.P. Borgers, and W.P.M.H. Heemels. Dynamic eventtriggered control: Tradeoffs between transmission intervals and performance. In IEEE Conference on Decision and Control, Los Angeles, U.S.A., pages 2764-2769, 2014.

[7] M.C.F. Donkers and W.P.M.H. Heemels. Output-based event-triggered control with guaranteed $\mathcal{L}_{\infty}$-gain and improved and decentralized event-triggering. IEEE Transactions on Automatic Control, 57(6):1362-1376, 2012.

[8] F. Forni, S. Galeani, D. Nešić, and L. Zaccarian. Event-triggered transmission for linear control over communication channels. Automatica, 50(2):490-498, 2014.

[9] A. Girard. Dynamic triggering mechanisms for event-triggered control. IEEE Transactions on Automatic Control (available on-line), 2015.

[10] R. Goebel, R.G. Sanfelice, and A.R. Teel. Hybrid dynamical systems. Princeton University Press, Princeton, U.S.A., 2012.

[11] W.P.M.H. Heemels, M.C.F. Donkers, and A.R. Teel. Periodic eventtriggered control for linear systems. IEEE Transactions Automatic Control, 58(4):847-861, 2013.

[12] W.P.M.H. Heemels, K.H. Johansson, and P. Tabuada. An introduction to event-triggered and self-triggered control. In CDC (IEEE Conference on Decision and Control) Maui, U.S.A., 2012.

[13] M. Mazo Jr and C. Ming. Asynchronous decentralized event-triggered control. Automatica, 50(12):3197-3203, 2014.

[14] H.K. Khalil. Nonlinear systems. Prentice-Hall, Englewood Cliffs, New Jersey, U.S.A., 3rd edition, 2002.

[15] M. Krichman, E.D. Sontag, and Y. Wang. Input-output-to-state stability. SIAM Journal on Control and Optimization, 39(6):18741928,2001

[16] M. Mazo and P. Tabuada. Decentralized event-triggered control over wireless sensor/actuator networks. In IEEE Transactions on Automatic Control, Special Issue on Wireless Sensor and Actuator Networks, volume 56, pages 2456-2461, 2011.

[17] C. De Persis, R. Sailer, and F. Wirth. Parsimonious event-triggered distributed control: a Zeno free approach. Automatica, 49(7):21162124, 2013.

[18] R. Postoyan, P. Tabuada, D. Nešić, and A. Anta. A framework for the event-triggered stabilization of nonlinear systems. IEEE Transactions on Automatic Control, 60(4):982-996, 2015.

[19] A. Seuret, C. Prieur, and N. Marchand. Stability of nonlinear systems by means of event-triggered sampling algorithms. IMA Journal of Mathematical Control and Information, 31(3):415-433, 2013.

[20] P. Tabuada. Event-triggered real-time scheduling of stabilizing control tasks. IEEE Transactions on Automatic Control, 52(9):1680-1685, 2007.

[21] P. Tallapragada and N. Chopra. Event-triggered decentralized dynamic output feedback control for LTI systems. IFAC Workshop on Distributed Estimation and Control in Networked Systems, 3(1):31-36, 2012.

[22] P. Tallapragada and N. Chopra. Decentralized event-triggering for control of nonlinear systems. IEEE Transactions on Automatic Control, 59(12):3312-3324, 2014.

[23] M. Velasco, J. Fuertes, and P. Marti. The self triggered task model for real-time control systems. 24th IEEE Real-Time Systems Symposium, pages 67-70, 2003.

[24] X. Wang and M.D. Lemmon. Self-triggered feedback control systems with finite-gain $\mathcal{L}_{2}$ stability. IEEE Transactions on Automatic Control, 45:452-467, 2009. 